\title{
ORIGINAL ARTICLE RECQ1 helicase is involved in replication stress survival and drug resistance in multiple myeloma
}

\author{
E Viziteu ${ }^{1}$, B Klein ${ }^{1,2,3}$, J Basbous ${ }^{1}$, Y-L Lin ${ }^{1}$, C Hirtz ${ }^{4}$, C Gourzones ${ }^{1}$, L Tiers ${ }^{4}$, A Bruyer ${ }^{1}$, L Vincent ${ }^{5}$, C Grandmougin ${ }^{1}$, A Seckinger ${ }^{6,7}$, \\ H Goldschmidt ${ }^{6,7}$, A Constantinou ${ }^{1}$, P Pasero ${ }^{1}$, D Hose ${ }^{6,7}$ and J Moreaux ${ }^{1,2,3}$
}

Multiple myeloma (MM) is a plasma cell cancer with poor survival, characterized by the expansion of multiple myeloma cells (MMCs) in the bone marrow. Using a microarray-based genome-wide screen for genes responding to DNA methyltransferases (DNMT) inhibition in MM cells, we identified RECQ1 among the most downregulated genes. RecQ helicases are DNA unwinding enzymes involved in the maintenance of chromosome stability. Here we show that RECQ1 is significantly overexpressed in MMCs compared to normal plasma cells and that increased RECQ1 expression is associated with poor prognosis in three independent cohorts of patients. Interestingly, RECQ1 knockdown inhibits cells growth and induces apoptosis in MMCs. Moreover, RECQ1 depletion promotes the development of DNA double-strand breaks, as evidenced by the formation of 53BP1 foci and the phosphorylation of ataxia-telangiectasia mutated (ATM) and histone variant H2A.X (H2AX). In contrast, RECQ1 overexpression protects MMCs from melphalan and bortezomib cytotoxicity. RECQ1 interacts with PARP1 in MMCs exposed to treatment and RECQ1 depletion sensitizes MMCs to poly(ADP-ribose) polymerase (PARP) inhibitor. DNMT inhibitor treatment results in RECQ1 downregulation through miR-203 deregulation in MMC. Altogether, these data suggest that association of DNA damaging agents and/or PARP inhibitors with DNMT inhibitors may represent a therapeutic approach in patients with high RECQ1 expression associated with a poor prognosis.

Leukemia (2017) 31, 2104-2113; doi:10.1038/leu.2017.54

\section{INTRODUCTION}

Multiple myeloma (MM) is the second most common hematologic malignancy. It is characterized by the accumulation of malignant plasma cells (MMCs) within the bone marrow (BM). MM is a genetically complex disease characterized by altered karyotypes including chromosomal translocations, aneuploidies and DNA copy-number variations. ${ }^{1}$ In addition to genetic changes, intraclonal heterogeneity of MMCs was recently described as a new level of complexity in MM pathophysiology. ${ }^{2-7}$ Epigenetic events could also participate in disease progression and treatment resistance. ${ }^{8}$ DNA methyltransferase (DNMT) inhibitors and histone deacetylases inhibitors are now being used in the treatment of several hematologic malignancies including $\mathrm{MM} .^{9-15}$

Recently, we initiated a microarray-based genome-wide screen for genes responding to DNMT inhibition in our large cohort of MM cell lines. ${ }^{16,17}$ We identified RECQ1 among the genes downregulated by DNMTi and associated with the worst prognostic value in MM patients. ${ }^{17}$ RECQ helicases are a family of DNA unwinding enzymes involved in the maintenance of chromosome stability. ${ }^{18}$ Several functions have been attributed to RECQ proteins, including roles in stabilization and repair of damaged DNA replication forks, homologous recombination and DNA damage checkpoint signaling. ${ }^{19}$ Mutations in three of the five human RecQ helicases, BLM, WRN and RECQL4, are associated with genetic disorders, respectively, with Bloom's, Werner and Rothmund-Thomson syndromes, which are characterized by chromosomal instability, premature aging and increased susceptibility to cancer. $^{19}$ RECQ1 promotes recovery of stalled replication forks. ${ }^{20-22}$ Furthermore, RECQ1 accumulates at replication origins in unperturbed cells and at common fragile sites where replication forks have stalled upon cellular exposure to replication inhibitors. $^{23}$ The role of RECQ1 in replication stress response could be especially important for cancer cells. ${ }^{24}$ RECQ1 is highly expressed in various types of solid tumors. ${ }^{25-28}$ RECQ1 silencing in cancer cells resulted in mitotic catastrophe and prevented tumor growth in murine models. ${ }^{24,28,29}$ Furthermore, RECQ1 depletion induces mitotic cell death in a wide range of solid cancer cell lines but not in normal fibroblasts. ${ }^{24}$ In glioblastoma cell lines, depletion of RECQ1 results in a significant reduction of cellular proliferation, spontaneous $\mathrm{YH} 2 \mathrm{AX}$ foci formation and hypersensitivity to treatments. ${ }^{25}$ The ability of RECQ1 to support replication recovery could be particularly important for cancer cells. RECQ1 binds G4 motifs in the promoter of genes that are downregulated upon RECQ1 silencing. ${ }^{30}$ Recent data suggest that RECQ1 could enhance the expression of genes involved in cell migration, invasion and metastasis in solid cancers. ${ }^{30}$ Accordingly, RECQ1 depletion in breast cancer cell line resulted in a significant inhibition of migration and invasion. Collectively, all these data underline that RECQ1 could play a role in MM pathophysiology and drug resistance with the hallmark feature of oncogene-induced DNA replication stress. $y$-histone variant H2A.X (H2AX) analysis demonstrated that MMCs have constitutive ongoing DNA damage. ${ }^{31,32}$ High chromosomal instability and replicative stress defines a subgroup of MM patients with poor outcome. ${ }^{33,34}$ Furthermore,

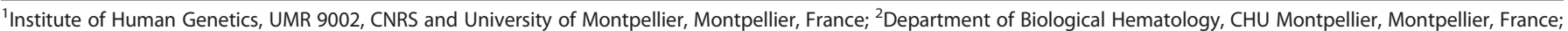

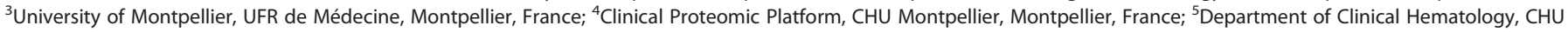

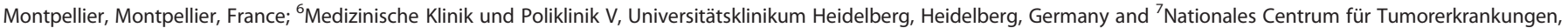

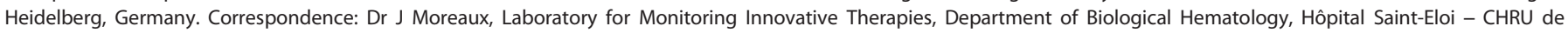
Montpellier, IGH - Institute of Human Genetics, UMR 9002, CNRS and University of Montpellier, 80, av. Augustin Fliche, Montpellier 34295, France. E-mail: jerome.moreaux@igh.cnrs.fr

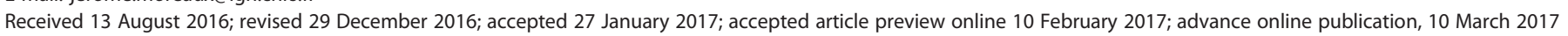


despite significant advances in MM treatment, including proteasome inhibitors, immunomodulatory agents and novel epigenetictargeted treatments, that significantly prolonged the median survival, the majority of MM patients relapse with the development of drug resistance. ${ }^{35}$ Therefore, we intent to explore the impact of RECQ1 in MM pathophysiology.

\section{MATERIALS AND METHODS}

Human myeloma cell lines (HMCLs)

XG1, XG2, XG3, XG4, XG5, XG6, XG7, XG10, XG11, XG12, XG13, XG14, XG16, XG19, XG20 and XG21 HMCLs were obtained as previously described. ${ }^{36}$ AMO-1, LP1, L363, U266, OPM2 and SKMM2 were purchased from DSMZ (Braunschweig, Germany) and RPMI8226 from ATTC (Rockville, MD, USA). HMCLs were authenticated according to their short tandem repeat profiling and their gene expression profiling using Affymetrix U133 plus 2.0 microarrays deposited in the ArrayExpress public database under accession numbers E-TABM-937 and E-TABM-1088. ${ }^{36}$

\section{Primary multiple myeloma cells}

BM samples were collected after patients' written informed consent in accordance with the Declaration of Helsinki and institutional research board approval from Heidelberg and Montpellier University hospital. In particular, BMs were collected from 206 patients treated with high-dose melphalan and autologous stem cell transplantation and this cohort is termed in the following Heidelberg-Montpellier cohort. ${ }^{37}$ Patients' MMCs were purified using anti-CD138 MACS microbeads (Miltenyi Biotech, Bergisch Gladbach, Germany) and their gene expression profile obtained using Affymetrix U133 plus 2.0 microarrays as described (E-MTAB-372). ${ }^{37}$ The structural chromosomal aberrations, including $\mathrm{t}(4 ; 14)(\mathrm{p} 16.3 ; \mathrm{q} 32.3)$ and $\mathrm{t}(11 ; 14)$ (q13;q32.3), as well as numerical aberrations including $17 \mathrm{p} 13$ and 1 q21 gain, were assayed by fluorescence in situ hybridization (iFISH). We also used publicly available Affymetrix gene expression profile (Gene Expression Omnibus, GSE2658) of a cohort of 345 purified MMC from previously untreated patients from the University of Arkansas for Medical Sciences (UAMS, Little Rock, AR, USA), termed in the following UAMS-TT2 cohort. $^{38}$ We also used total therapy 3 cohort (UAMS-TT3; $n=158$; E-TABM-1138 $)^{39}$ and data of 152 relapsed MM patients subsequently treated with bortezomib (GSE9782) from the study by Mulligan et al. ${ }^{40}$

\section{Cell growth assay, cell cycle and apoptosis analyses}

HMCLs were cultured for 4 days in 96-well flat-bottom microtiter plates in RPMI 1640 medium, 10\% FCS and $2 \mathrm{ng} / \mathrm{ml} \mathrm{IL-6}$ (control medium) in the presence of various compounds. Cell growth was evaluated by quantifying intracellular ATP amount with a Cell Titer Glo Luminescent Assay (Promega, Madison, WI, USA) using a Centro LB 960 luminometer (Berthold Technologies, Bad Wildbad, Germany). Doxycycline $(1 \mathrm{mg} / \mathrm{ml})$ was added to induce shRECQ1 expression. The cell cycle was assessed using 49,6-diamidino-2-phenylindole (DAPI) staining (Sigma-Aldrich, Munich, Germany), incubation with bromodeoxyuridine (BrdU) for $1 \mathrm{~h}$, and labeling with an anti-BrdU antibody (APC BrdU flow kit, BD Pharmingen, Le Pont De Claix, France) as described. ${ }^{41}$ Apoptotic cells were detected using phycoerythrin-conjugated Annexin V (PE-annexin V, BD Pharmingen). Apoptosis was measured using caspase-3/7 and caspase- 9 luminescence Assay (Caspase Glo Assay (Promega, Madison, WI, USA)) according to the manufacturer's protocol.

\section{RECQ1 depletion in primary MM cells}

BM of patients presenting with previously untreated MM $(n=7)$ at the university hospital of Montpellier was obtained after patients' written informed consent in accordance with the Declaration of Helsinki and agreement of the Montpellier University Hospital Centre for Biological Resources (DC-2008-417). Mononuclear cells were transfected with shRECQ1 or shRNA scramble lentivirus and MMC cytotoxicity evaluated using anti-CD138-phycoerythrin monoclonal antibody (Immunotech, Marseille, France) as described. ${ }^{17}$

\section{DNA repair foci-immunofluorescence microscopy}

After deposition on slides using a Cytospin centrifuge, cells were fixed with $4 \%$ paraformaldehyde, permeabilized with $0.5 \%$ Triton in phosphatebuffered saline (PBS) and saturated with $5 \%$ bovine milk in PBS. The rabbit
anti-53BP1 antibody (clone NB100-304, Novus Biologicals, Cambridge, UK) and the mouse anti- $\gamma \mathrm{H} 2 \mathrm{AX}$ (Ser139) antibody (clone JBW301, Merck Millipore, Darmstadt, Germany) were diluted $1 / 300$ and $1 / 100$, respectively, in $5 \%$ bovine milk in PBS, and deposited on cytospins for 90 min at room temperature. Slides were washed twice and antibodies to rabbit or mouse immunoglobulins conjugated to alexa 488 (diluted $1 / 500$ in $5 \%$ bovine milk in PBS) were added for $45 \mathrm{~min}$ at room temperature. Slides were washed and mounted with Vectashield and 1\% DAPI. Images and fluorescence were captured with a ZEISS Axio Imager Z2 microscope ( $\times 63$ objective), analyzed with Metafer (version3.6, company, town state) and ImageJ software. The number of 53BP1 and $\mathrm{\gamma H} 2 \mathrm{AX}$ foci was counted in at least 300 nuclei.

Supplementary informations concerning methodology are included in Supplementary experiment procedures.

\section{RESULTS}

RECQ1 is overexpressed in MMCs

RECQ1 expression was analyzed in normal bone marrow plasma cells (BMPCs), primary myeloma cells from newly diagnosed patients (MMCs) and HMCLs using Affymetrix microarrays. RECQ1 gene was significantly overexpressed in MMCs of patients (median $=1499$; range: $251-6488$ ) compared to normal BMPCs (median $=841$; range: $523-1519 ; P<0.001$ ). The expression was even higher in HMCLs (median=3233; range: 811-5873) compared to primary MMCs or BMPCs $(P<0.001$; Figure 1a). RECQ1 expression was confirmed at protein level in HMCLs, in patients' primary MMCs (Figure 1b). RECQ1 expression was significantly higher in the poor prognosis proliferation group $(P<0.01)$ and in the CD1 group $(P<0.05)$, and downregulated in the hyperdiploid MM subgroup ${ }^{42}$ (Supplementary Figure S1A). RECQ1 overexpression in MMCs compared to normal $B$ and plasma cells was also validated by $\mathrm{qPCR}$ and western blot (Supplementary Figure S1B and Figure 1b). A high RECQ1 expression in MMCs could predict for both shorter event-free (EFS) and overall survival (OS) in the Heidelberg-Montpellier cohort of 206 previously untreated patients (Figure 1c). These patients could be split in two groups according to RECQ1 expression and patients' survival using the Maxstat algorithm. ${ }^{16,43}$ A group encompassing $17.5 \%$ of patients with the highest RECQ1 expression in MMCs had reduced EFS (median $=22.7$ months) and OS (median $=73.3$ months) compared to the remaining patients with lower RECQ1 expression (median $\mathrm{EFS}=34.2$ months and median $\mathrm{OS}=92$ months, $P \leqslant 0.01$ ) (Figure 1c). A high RECQ1 expression in MMCs was likewise associated with a poor prognosis in the independent UAMS-TT2 (Supplementary Figure S2A, $P<0.001$ ) and UAMS-TT3 cohorts of patients (Supplementary Figure $\mathrm{S} 2 \mathrm{~B}, P=0.001$ ). Of note, $R E C Q 1$ was significantly overexpressed in patients with del17p, del13 and 1q21 gain (Supplementary Figure S2C), but no correlation was observed with $t(4 ; 14)$ and $t(11 ; 14)$ translocations. The frequency of patients with age $\geqslant 65$ years, high $\beta$-2-microglubuline or high $\mathrm{C}$-reactive protein levels were significantly increased in patients with high RECQ1 expression ( $P<0.05$; Supplementary Table S1). No significant correlation between RECQ1 expression and MMC plasma cell labeling index was found (Supplementary Figure S3).

Interestingly, gene set expression analysis of patients with high RECQ1 expression highlighted a significant enrichment of genes involved in embryonic stem cells transcriptional program and overexpressed in plasmablasts, compared to BM mature plasma cells (Supplementary Figure S4A and Supplementary Tables S2 and S3). In contrast, patients with low RECQ1 expression are characterized by a significant enrichment of genes overexpressed in $\mathrm{TACl}^{\text {high }} \mathrm{MMCs}$ and involved in cytokine/cytokine receptor interactions linked with a mature plasma cell gene signature and BM microenvironment dependence ${ }^{44}$ (Supplementary Figure S4B and Supplementary Tables S4 and S5). Altogether, these data indicate that RECQ1 is significantly overexpressed in MM cells in association with a plasmablastic gene signature and a poor prognosis. 
a

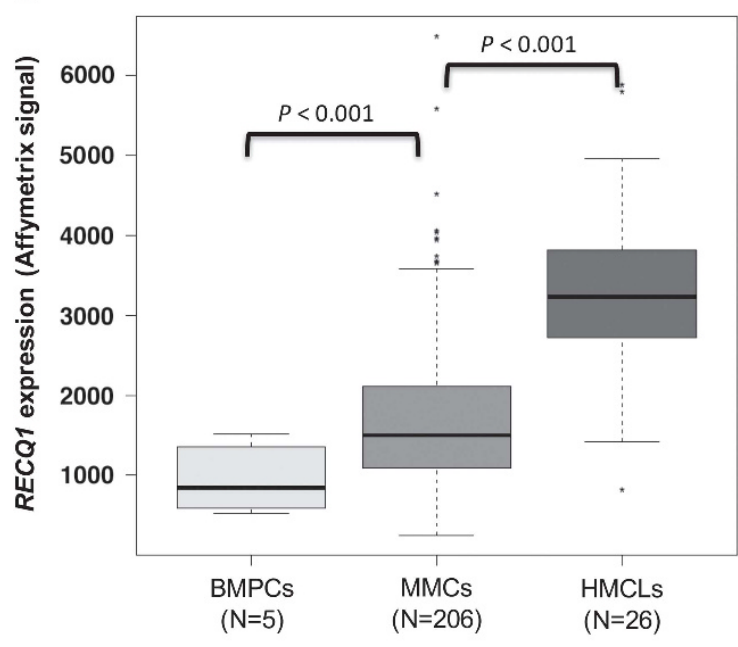

b

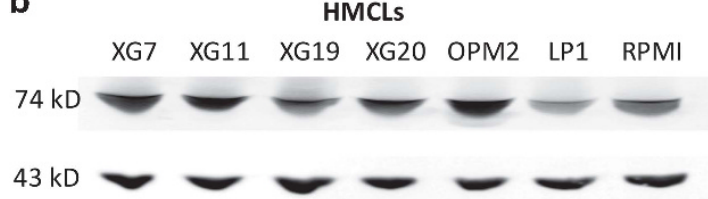

c

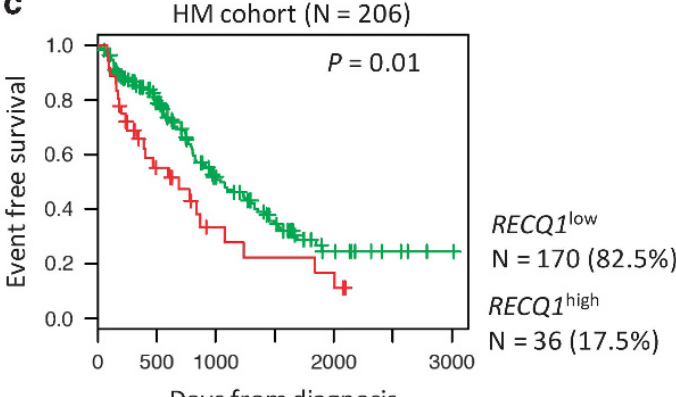

Days from diagnosis

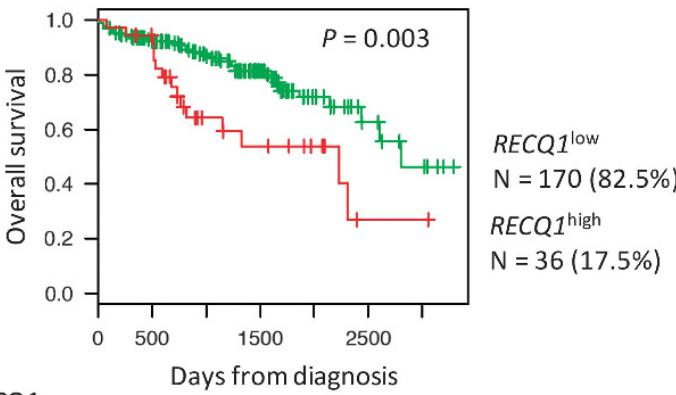

RECQ1

Beta Actin
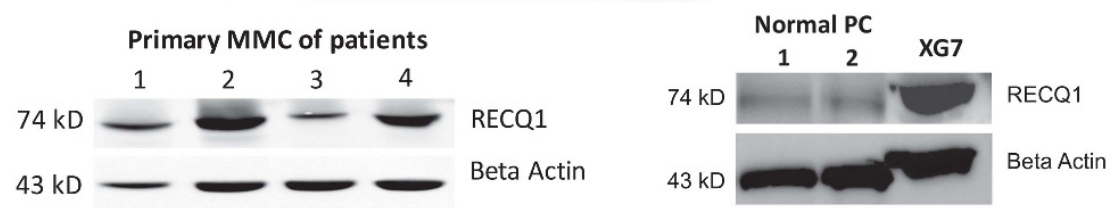

Figure 1. RECQ1 expression in MM. (a) RECQ1 gene expression in normal BMPCs, patients' MMCs and HMCLs. Data are MAS5-normalized Affymetrix signals (U133 plus 2.0 microarrays). Statistical difference was tested using a Student $t$-test. (b) Western blot showing RECQ1 expression in seven different HMCLs, normal in vitro-generated plasma cells ${ }^{62}$ and purified primary MMCs of four patients. (c) High RECQ1 expression in MMCs could predict for shorter overall and event-free survival. Patients of the Heidelberg-Montpellier cohort $(N=206)$ were ranked according to increasing RECQ1 expression and a maximum difference in OS and EFS was obtained using the Maxstat R function.

RECQ1 depletion in MMCs affects cell division and induces apoptosis

To investigate the functional significance of RECQ1 overexpression in multiple myeloma cells, XG19, LP1 and XG7 HMCLs were transduced with a doxycycline-inducible lentivirus containing a RECQ1 shRNA. Doxycycline treatment induced a $68-87 \%$ reduction of RECQ1 expression at RNA level $(P<0.01$; Supplementary Figure S5) and a 70-90\% reduction at the protein level $(P<0.01$; Figures $2 a$ and b). RECQ1 depletion induced a progressive death of XG19, LP1 and XG7 TR-shRNA RECQ1 cells, which started 3-4 days after doxycycline addition (Figure 2c, $P=0.001$ and Supplementary Figure S6A). Doxycycline marginally affected the growth of control HMCL-TRs (Figure 2c and Supplementary Figure S6A). Remarkably, RECQ1 depletion induced apoptosis in HMCL-TR-shRECQ1, which initiated on day 3 and increased on day 7, with 79, 53 and 38\% of Annexin-V-positive XG19, LP1 and XG7 TR-shRECQ1 cells, respectively (Supplementary Figure S6B, $P<0.05)$. Apoptosis induced by the depletion of RECQ1 was associated with activation of caspase- $9,-3$ and -7 (Figure $2 d$ ) and could be rescued by adding the Quinoline-Val-Asp-Difluorophenoxymethylketone pan-caspase inhibitor (Supplementary Figure S7). RECQ1 depletion also affected the cell cycle distribution of surviving cells with a blockage of BrdU incorporation and an increase in the $G_{1}$ and $G_{2} / M$ phases $(P<0.05$; Figure 3a, $P<0.01$ and Supplementary Figures S8A and B). These phenotypes were reproduced after RECQ1 suppression using a second
shRNA with different targeting sequence (Supplementary Figures S9A, B, C and D) and rescued by the ectopic expression of a shRNAresistant RECQ1 gene (Supplementary Figures S10A, B and C).

To confirm that RECQ1 is also required for the survival of primary MMCs from patients, BM samples containing malignant MMCs were transduced ${ }^{45,46}$ with shRECQ1 lentiviruses and primary MM cells were cultured with their BM environment and recombinant IL-6 as described. ${ }^{17}$ RECQ1 depletion significantly reduced the median number of viable myeloma cells by $56 \%$ $(P=0.004 ; N=7)$ compared to scramble shRNA (Figure $3 \mathrm{~b}$ ). Of interest, the normal BM non-myeloma cells were not significantly affected by the shRNA (Figure 3b). RECQ1 expression downregulation after transduction was validated in primary MMC of patient using real-time PCR (Supplementary Figure S11). We therefore conclude that viability of MM cell lines and primary MM cells depends on high RECQ1 expression levels.

RECQ1-deficient MMCs show spontaneous DNA breaks and activate the DNA damage response (DDR)

Given the known role of RECQ1 in DNA replication and DDR, ${ }^{21,47}$ the effect of RECQ1 depletion on spontaneous DNA damage was investigated. Interestingly, RECQ1 depletion in XG19 HMCLs induced DNA strand breaks as indicated by the alkaline comet assay that detect both single- and double-strand breaks (Figure 4a and Supplementary Figure S12). Indeed, a significant increase in comet tail length and moment was found in XG19-TR-shRECQ1 

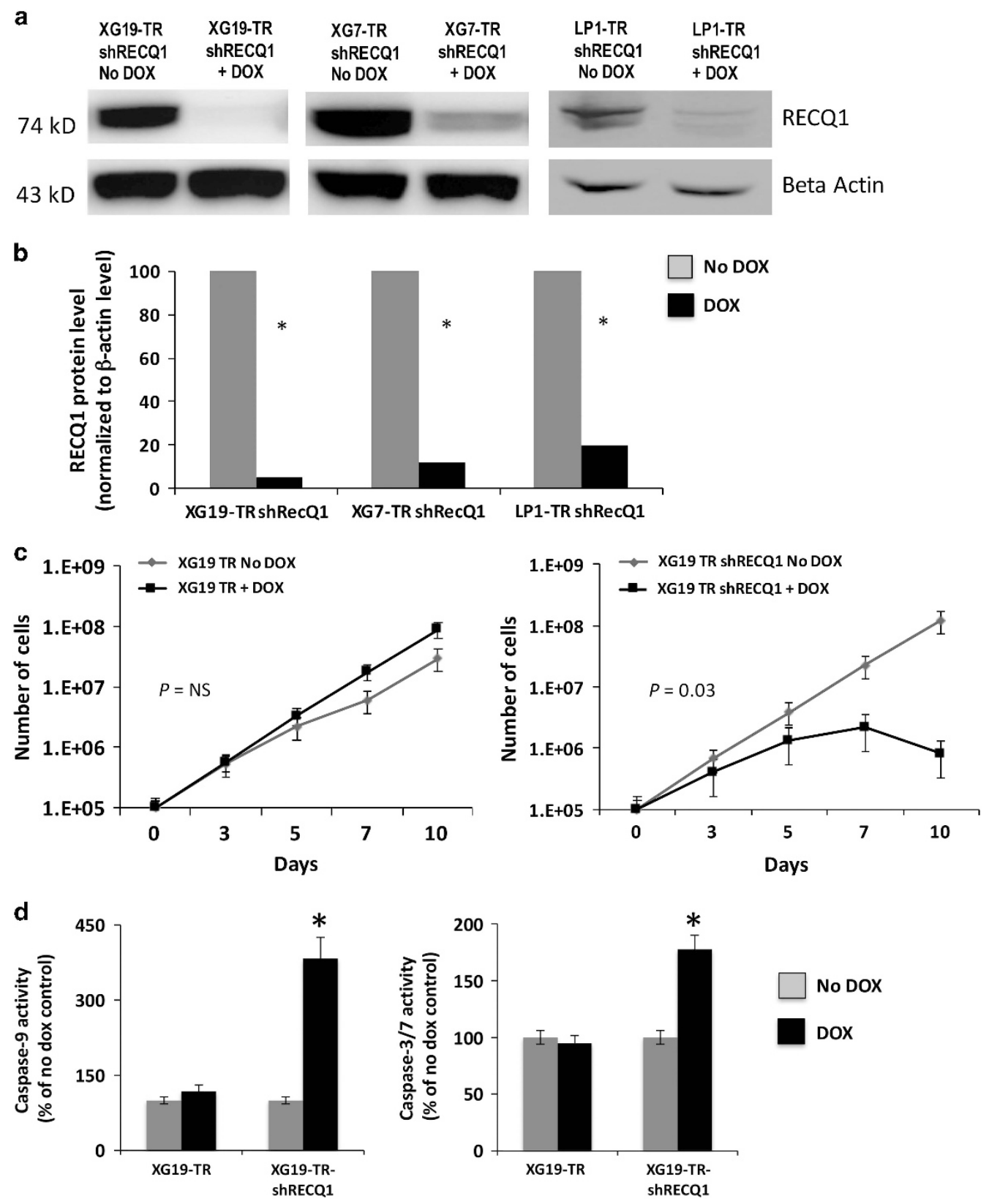

Figure 2. RECQ1 depletion in MMC results in cell growth inhibition and apoptosis induction. (a) Doxycycline-mediated inhibition of RECQ1 protein expression in three different HMCLs transduced with a RECQ1 shRNA inducible vector. Data are those of one experiment out of three. Protein load was assayed using $\beta$-actin quantification. (b) The expression of RECQ1 protein was quantified with ImageJ software, normalized to $\beta$-actin level, and data are the mean normalized values of RECQ1 expression. * indicates a significant decrease compared to doxycycline (dox)untreated cells using a Wilcoxon test for pairs $(P \leqslant 0.05)$ (three independent experiments). (c) Inducible depletion of RECQ1 delays myeloma cell growth. The HMCLs transduced with the TR lentivirus or with both TR and RECQ1 shRNA (shRECQ1) lentiviruses were exposed to doxycycline and cell viability analyzed by trypan blue assay. Results are those of one experiment representative of five. Statistical significance was tested using a Wilcoxon test for pairs. (d) Caspase activity was evaluated by luminescence assay in XG19-TR-shRECQ1 5 days after doxycycline treatment. The activity of caspase- 9 and caspase-3/7 was significantly elevated in RECQ1-depleted cells $(*$ indicates a $P$-value $<0.01)$. Data are those of one experiment representative of three. Statistical significance was tested using a Wilcoxon test for pairs.

cells treated with doxycycline compared to control cells $(P<0.001$, Figure 4a). RECQ1 depletion was also associated with the accumulation of DNA double-strand breaks, as evidenced by increased phosphorylation of the histone variant $\mathrm{H} 2 \mathrm{AX}$ and the accumulation of 53BP1 foci (Figures $4 \mathrm{~b}$ and $\mathrm{c}$ and Supplementary Figure S13A). These data were validated after RECQ1 suppression using a second shRNA with different targeting sequence (Supplementary Figure S13B). Finally, RECQ1 depletion induced a DDR associated with an increased phosphorylation of ataxiatelangiectasia mutated (ATM), Chk1, Chk2, P53 and H2AX
(Figure 4c). These phenotypes were rescued by the ectopic RECQ1 gene expression (Supplementary Figure S10D). Together, these data indicate that high RECQ1 levels protect MMCs from spontaneous intrinsic DNA damage.

Delayed progression of the replication forks upon RECQ1 depletion

Although it has been reported that RECQ1 plays a role in the maintenance of DNA replication forks, ${ }^{20}$ we next monitored the 


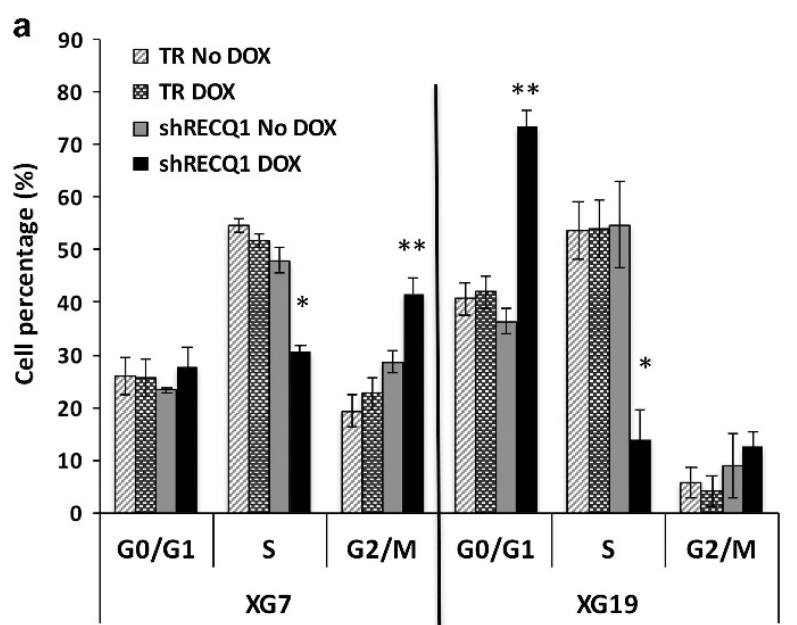

b
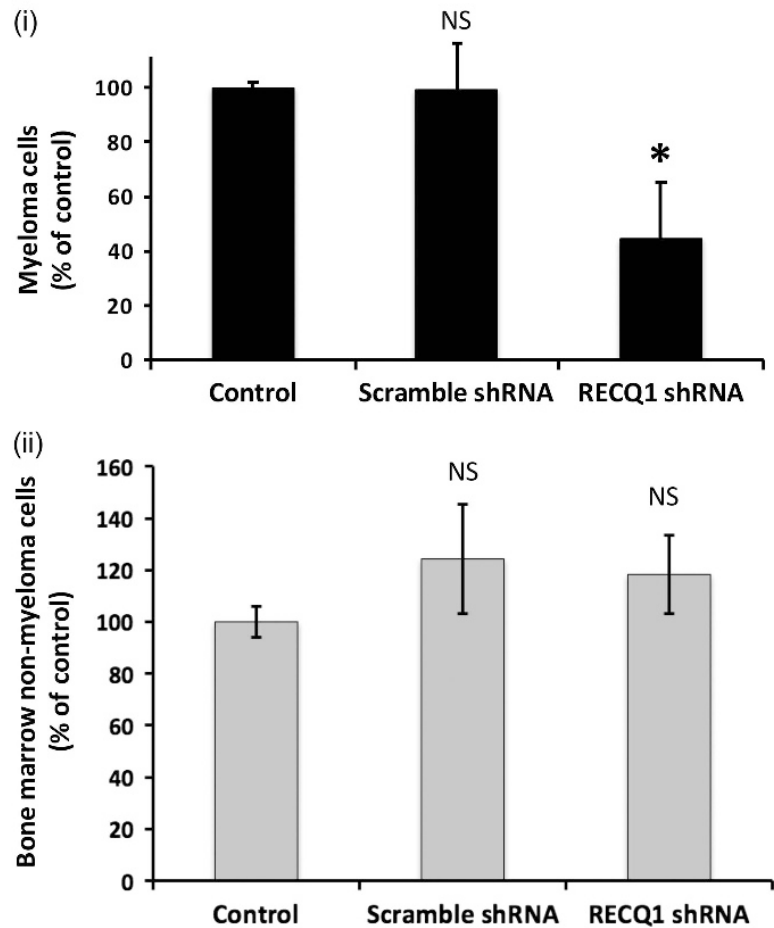

Figure 3. RECQ1 depletion induces defects in cell cycle progression of HMCL and mortality of primary MM cells from patients. (a) Cell cycle of RECQ1-depleted MM cell lines was analyzed by flow cytometry using BrdU incorporation and labeling with an anti-BrdU antibody and DAPI. Results are representative of five independent experiments. * indicates a significant decrease compared to doxycycline (dox)-untreated cells $(P<0.05$; Wilcoxon test for pairs). ** indicates a significant increase compared to dox-untreated cells $(P<0.05$, Wilcoxon test for pairs). (b) Mononuclear cells from seven patients with $\mathrm{MM}$ were transduced with shRECQ1 or shRNA scramble lentiviruses. At day 4 of culture, the viability and total cell counts were assessed and the percentage (i) $\mathrm{CD}_{138^{+}}$viable plasma cells and (ii) bone marrow non-myeloma cells were determined by flow cytometry. Results are median values of the numbers of myeloma cells in the culture wells. Results were compared with a Wilcoxon test for pairs. ${ }^{*}$ indicates a significant decrease compared to scramble shRNA $(P<0.01)$.

role of increased RECQ1 expression on fork progression in MM cells using DNA fiber analysis. To this end, HMCLs were pulselabeled with $10 \mu \mathrm{m}$ IdU and $100 \mu \mathrm{m}$ CldU and the length of CldU tracks was measured to estimate the distance covered by individual forks during the pulse. Interestingly, the length of CldU tracks was significantly shorter in RECQ1-depleted MMCs relative to control cells $(P<0.0001$; Figure $4 d)$. These data indicate that high RECQ1 levels promote the efficient progression of replication forks in MMCs and suggest that defective fork progression accounts for the increased spontaneous DNA damage in RECQ1deficient MMCs.

RECQ1 overexpression protects MMCs against melphalan and bortezomib

The results presented above indicate that the overexpression of RECQ1 in MMCs protects them from spontaneous DNA damage and replication stress. Since chemotherapy also induces DNA breaks, we next investigated whether RECQ1 overexpression protects MMCs against dexamethasone, bortezomib or melphalan (Figures 5a and d). Remarkably, the forced expression of RECQ1 in XG7 and XG19 HMCLs (Supplementary Figure S14) resulted in an increased resistance to melphalan and bortezomib-induced cell death, $(P<0.05$; Figures 5 a and $d)$, but had no significant effect on the sensitivity to lenalidomide (Supplementary Figure S15) and dexamethasone (data not shown). Furthermore, a BrdU incorporation assay demonstrated that RECQ1-overexpressing cells surviving after melphalan exposure are still able to proliferate, whereas control myeloma cells are irreversibly blocked in $G_{2} / M$ phase (Figure 5b and Supplementary Figures S16A and B). Interestingly, increased RECQ1 levels did not affect the number of $\gamma \mathrm{H} 2 \mathrm{AX}$ foci $24 \mathrm{~h}$ after melphalan treatment demonstrating that melphalan induced the same amount of DNA damages in control and myeloma cells overexpressing RECQ1 (Supplementary Figure S17). However, after $72 \mathrm{~h}$ of melphalan treatment, the comet assay revealed that cells overexpressing RECQ1 repair DNA breaks induced by melphalan more efficiently than control cells (Figure 5c).

Although RECQ1 overexpression is inhibited by DNMTi such as 5 -azacytidine, ${ }^{17}$ we next tested whether 5 -azacitidine treatment sensitize MMCs to melphalan-induced apoptosis. We found that both 5-azacytidine and the direct depletion of RECQ1 with shRNAs sensitized MMCs to melphalan (Supplementary Figures S18A-C). RECQ1 overexpression protects MMCs from bortezomib-induced toxicity (Figure $5 \mathrm{~d}$ ). These observations are consistent with the fact that high RECQ1 expression is associated with a poor prognosis in a cohort of patients at relapse treated by bortezomib monotherapy (Figure $5 \mathrm{~d}$ ). Altogether, these data indicate that RECQ1 plays a central role in the protection of MM cells against both spontaneous DNA damage and DNA lesions induced by chemotherapeutic agents such as bortezomib and melphalan.

\section{Identification of RECQ1-interacting proteins}

To identify the proteins that interact with RECQ1 in MMCs, we next immunoprecipitated RECQ1 from the chromatin fraction prepared from XG7 cells. Duplicate mass spectrometry analysis of the SDSPAGE lanes obtained from RECQ1 immunoprecipitation resulted in a total of 183 proteins after subtraction of the proteins commonly identified with the IgG control antibody (Supplementary Table S6). Among them, several proteins already described as RECQ1interacting partners were identified, including RPA, PARP1, Ku80, MSH2 and MSH6 ${ }^{\text {(refs }}$ 48,49) (Supplementary Table S6). We also observed a significant enrichment in proteins involved in RNA processing and splicing, transcription regulation, regulation of helicase activity and regulation of DDR (Supplementary Table S7), including PARP1, which is consistent with an earlier study. ${ }^{20}$

RECQ1 interacts with PARP1 and RECQ1 depletion sensitizes MMCs to PARPi

RECQ1 and PARP1 are involved in the control of DNA repair, telomere maintenance and replicative stress responses. ${ }^{50}$ 
a

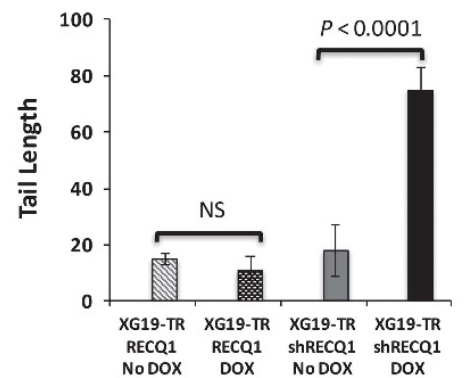

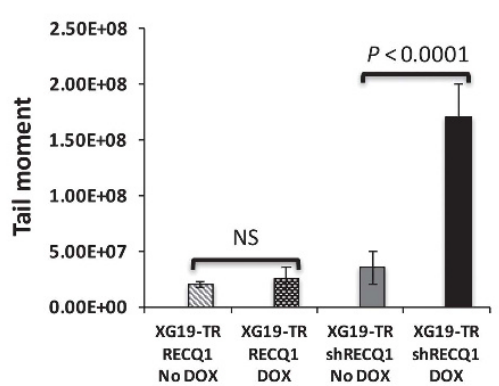

$\begin{array}{cccc}\text { NoDOX DOX } & \text { NoDOX } & \text { DOX }\end{array}$
C

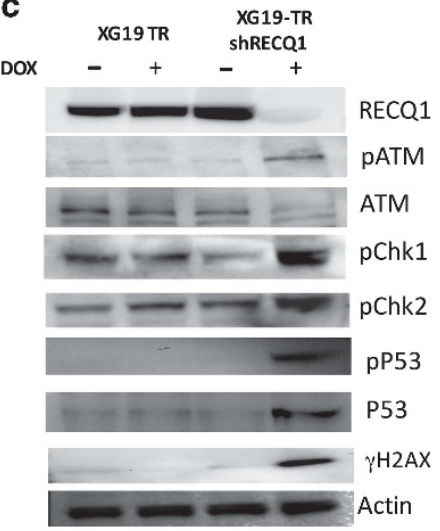

b

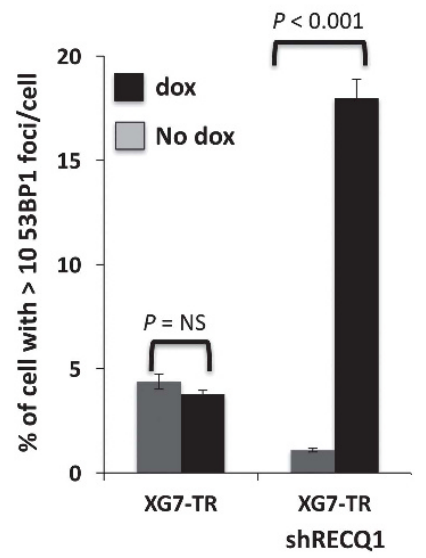

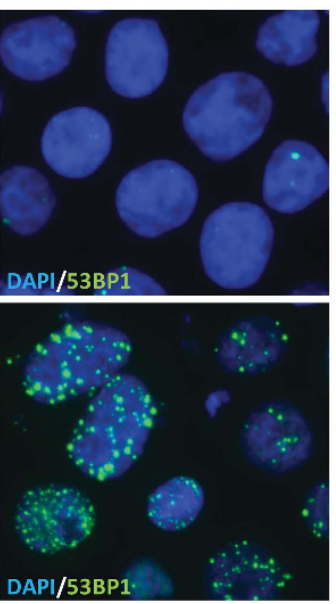

XG7 TR

ShRNA RECQ1

No DOX

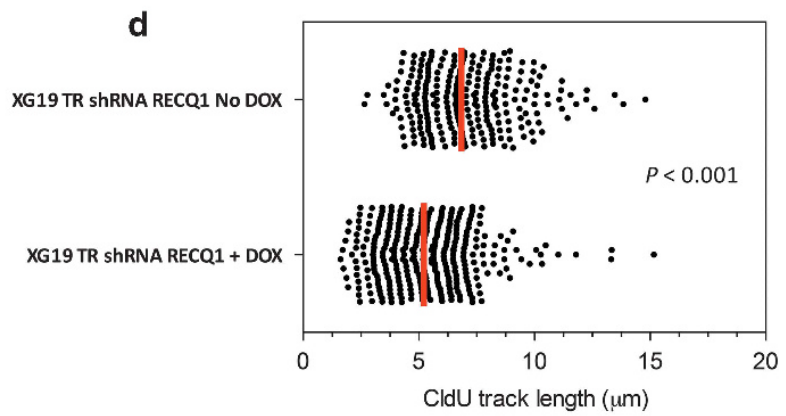

Figure 4. RECQ1 depletion induces DDR in MM cells. (a) Alkaline comet assay was used to analyze DNA breaks formation after RECQ1 depletion in XG19 cell line. Tail length and tail moment (fraction of total DNA in the tail $\times$ tail length) were analyzed with ImageJ software. Data are calculated from measurement of 50 comets for each sample. Statistical difference was tested using a Student $t$-test. (b) $53 \mathrm{BP} 1 \mathrm{staining}$ was used as marker for DNA damage. The number of 53BP1 foci found in each cell was enumerated 3 days after doxycycline treatment. At least 300 cells were counted for each group. The percentage of cells with more than 10 53BP1 foci per cell is displayed in the histograms. Statistical difference was tested using a Student $t$-test. (c) HMCL-TR-shRECQ1 cells were cultured for 7 days with or without doxycycline (dox) and protein detection was assayed using western blot analysis. Membranes were stained with anti-phospho-ATM, anti-ATM, anti-phospho-p53 (Ser 15 or Ser20) and anti-p53, anti-Phospho-Chk2, anti-Chk2 and anti- $\gamma \mathrm{H} 2 \mathrm{AX}$. A mouse monoclonal anti- $\beta$-actin antibody was used as control. (d) XG19-TR-shRECQ1 cell line was treated or not with doxycycline for 5 days and replication fork progression was determined by DNA fiber spreading after double labeling with IdU and CldU. Statistical difference was tested using a Student $t$-test.

To further understand the mechanisms of DNA damage induced by RECQ1 depletion in MMCs and validate proteomic results, we analyzed the interactions between PARP1 and RECQ1 in XG7 nuclear extracts. In agreement with mass spectrometry results, we found that PARP1 immunoprecipitates with RECQ1 (Supplementary Figure S19). Of note, the amount of PARP1 bound to RECQ1 was increased after treatment of MMCs with melphalan and bortezomib (Supplementary Figures S19A and B). Given the association of RECQ1 and PARP1 and the clinical development of poly(ADP-ribose) polymerase (PARP) inhibitors in cancer, we next investigated how RECQ1 depletion affects the sensitivity of MMCs to PARP inhibitor. Cells were pulsed with doxycycline for 2 days and treated with increasing PJ34 hydrochloride concentrations for 4 days. RECQ1 depletion significantly sensitized XG7 and XG19 HMCLs to PARPi-induced apoptosis (Figures $6 a$ and b).

DNMTi induces miR-203 expression and targets RECQ1 expression in MMCs

RECQ1 expression was recently described as one of the predicted targets of miR-203 in tongue squamous cell carcinoma. ${ }^{27}$ Since aberrant methylation of miR-203 promoter region was reported in MMCs but not in normal plasma cells, ${ }^{51}$ this prompted us to investigate the expression of miR-203 in MMCs exposed to 5-azacytidine. Remarkably, we observed that 5-azacytidine treatment significantly induced miR-203 expression in HMCLs and a concomitant downregulation of RECQ1 (Figure 7a). Moreover, treatment of XG7 and XG19 HMCLs with a specific anti-miR-203 miRNA inhibitor resulted in a significant increase of RECQ1 expression (Figure 7b). Altogether, these data suggest that the epigenetic downregulation of miR-203 in MMCs allows RECQ1 overexpression and increased resistance to melphalan and bortezomib.

\section{DISCUSSION}

Here we show that RECQ1 plays a central role in MM biology and in the resistance of MMCs to chemotherapy. Recent studies have pointed out a critical role of RECQ1 in the process of DNA replication, repair and transcription. ${ }^{52}$ Our data confirm and extend this role to HMCLs and to primary MMCs. In MM, a subset of patients is characterized by chromosomal instability, replicative stress and adverse prognosis. ${ }^{34}$ Furthermore, MYC dysregulation triggers replicative stress and DNA damage in MM cells. ${ }^{34}$ In HMCLs, we found that RECQ1 depletion induced a dramatic increase of spontaneous DNA damage, illustrated by the presence of ssDNA breaks in virtually all cells analyzed with the comet assay. Spontaneous DNA double-strand breaks (DSBs) were also 

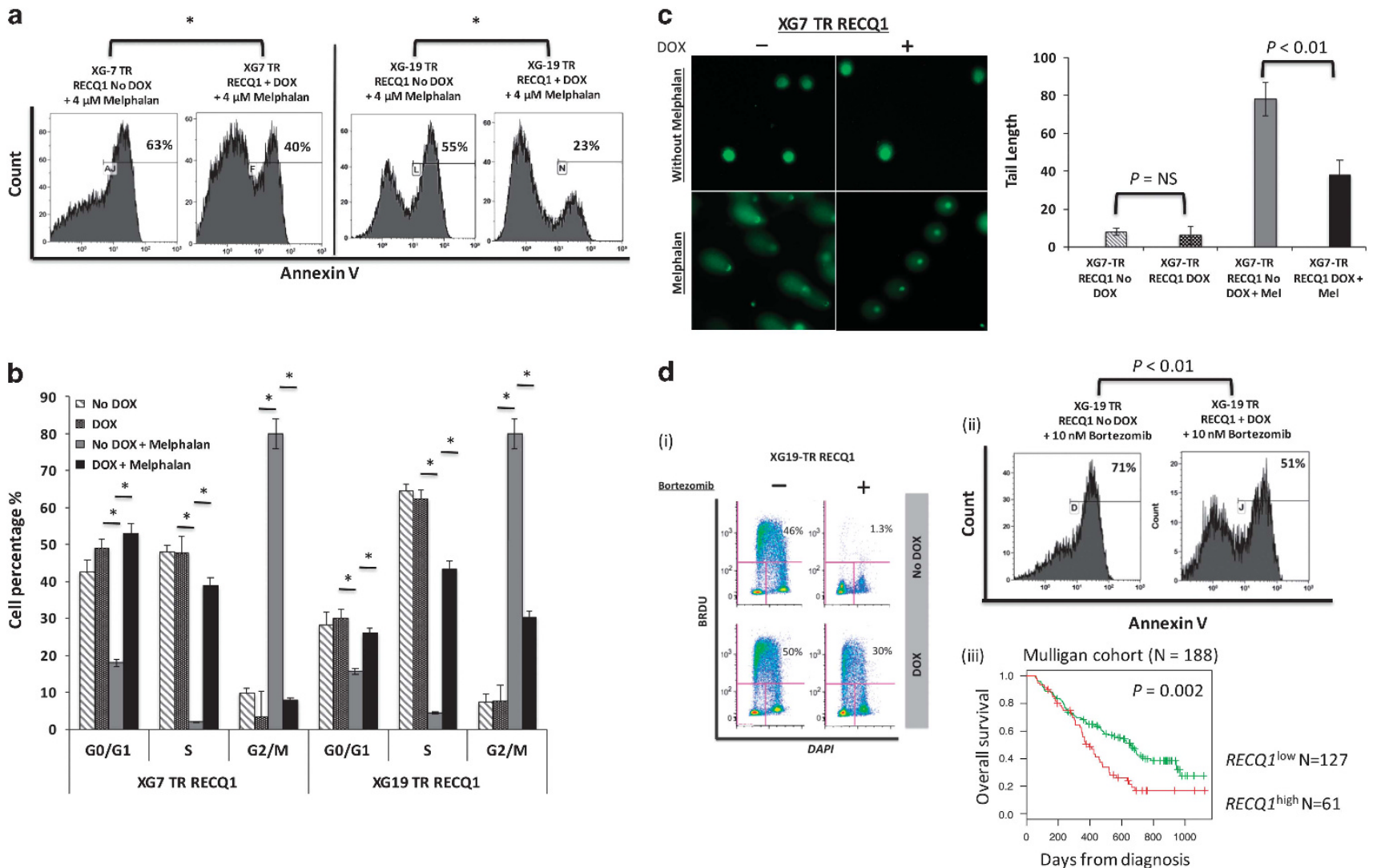

Figure 5. Inducible overexpression of RECQ1 protects MMC from melphalan and bortezomib-induced toxicity. (a) HMCL apoptosis induction was assessed with Annexin V, by flow cytometry after treatment or not with $4 \mu \mathrm{m}$ of melphalan for 4 days. ${ }^{*}$ indicates a significant decrease compared to doxycycline-untreated cells using a Wilcoxon test for pairs $(P \leqslant 0.05)$. Results are those of one experiment representative of five. (b) HMCL-TR-RECQ1 cells were treated or not with doxycycline. Cell cycle of non-apoptotic cells was analyzed by flow cytometry using BrdU incorporation and labeling with an anti-BrdU antibody and DAPI after 3 days of treatment with $4 \mu \mathrm{m}$ of melphalan. Results are representative of five independent experiments. ${ }^{*}$ indicates a significant difference using a Wilcoxon test for pairs $(P<0.05)$. (c) MMC overexpressing or not RECQ1 were treated with $4 \mu \mathrm{m}$ of melphalan (Mel) for $72 \mathrm{~h}$ and analyzed using alkaline comet assay. Tail length and tail moment (fraction of total DNA in the tail $\times$ tail length) were analyzed with ImageJ software. Data are calculated from measurement of 50 comets for each sample. Results are representative of three independent experiments. Statistical difference was tested using a Student $t$-test. (d) XG19 MM cell line overexpressing or not RECQ1 were treated with $10 \mathrm{~nm}$ of bortezomib for 4 days. Cell cycle was analyzed by flow cytometry using BrdU incorporation and labeling with an anti-BrdU antibody and DAPI. Results are those of one experiment representative of three. Statistical difference was tested using a Wilcoxon test for pairs (i) and apoptosis induction was analyzed with Annexin V APC staining by flow cytometry. Results are those of one experiment representative of three. Statistical difference was tested using a Wilcoxon test for pairs (ii). High RECQ1 expression is associated with a shorter OS in a cohort of 188 patients at relapse treated with bortezomib monotherapy (Mulligan cohort $^{40}$ ) (iii).

detected by immunofluorescence using $\mathrm{Y}-\mathrm{H} 2 \mathrm{AX}$ and 53BP1 foci as markers of chromosome breaks. These breaks were accompanied with an impaired progression of replication forks and the persistence of surviving MMCs in the $G_{1}$ or $G_{2}$ phases of the cell cycle, followed by their progressive death. RECQ1 depletion also induced a massive death of patients' MMCs without poorly affecting the survival of non-tumor environment cells. Our data demonstrate that RECQ1 contributes to replication stress survival of MM cells.

In spite of effective therapeutic protocols developed in MM, drug resistance remains a major concern. We reported that RECQ1 overexpression was associated with drug resistance in MMC. RECQ1 plays a central role in the protection of $M M$ cells against DNA lesions induced by chemotherapeutic agents such as melphalan and bortezomib. Melphalan is an alkylating agent used in patients receiving high-dose chemotherapy and autologous hematopoietic stem cell transplantation and in nontransplantable patients in combination with other compounds. Bortezomib is a proteasome inhibitor used as a first-in-class drug in MM. Since, protein ubiquitination is essential in regulation and coordination of various pathways of DNA damage recognition, signaling and repair, ${ }^{53}$ proteasome inhibitors affect DNA repair. $^{54-56}$ The protective effect of RECQ1 overexpression against melphalan and bortezomib is associated with the poor prognosis of high RECQ1 expression in MM patients treated with high-dose melphalan and autologous hematopoietic stem cell transplantation (Figure 1c and Supplementary Figures S2A and B) and in relapsing patients treated with bortezomib monotherapy (Figure 5d). Among the RECQ1-interacting proteins, we identified several proteins involved in DNA repair pathways, including PARP1, RPA, Ku80, MSH2 and MSH6. Regression of replication fork appears as a pivotal response mechanism to replication stress and occurs in $15-40 \%$ of the forks after exposure to sublethal doses of Top1 inhibitors. Interestingly, this process is regulated by RECQ1 and PARP $1 .{ }^{20}$ PARP activity is dispensable in the formation of reversed forks but is important to maintain and protect them from the counteracting activity of RECQ1 that would cause the restart of reversed forks, leading to DSB formation. ${ }^{20}$ PARP was shown to signal the presence of lesions on the template and to inhibit RECQ1 locally, thereby restraining the restart of reversed forks 

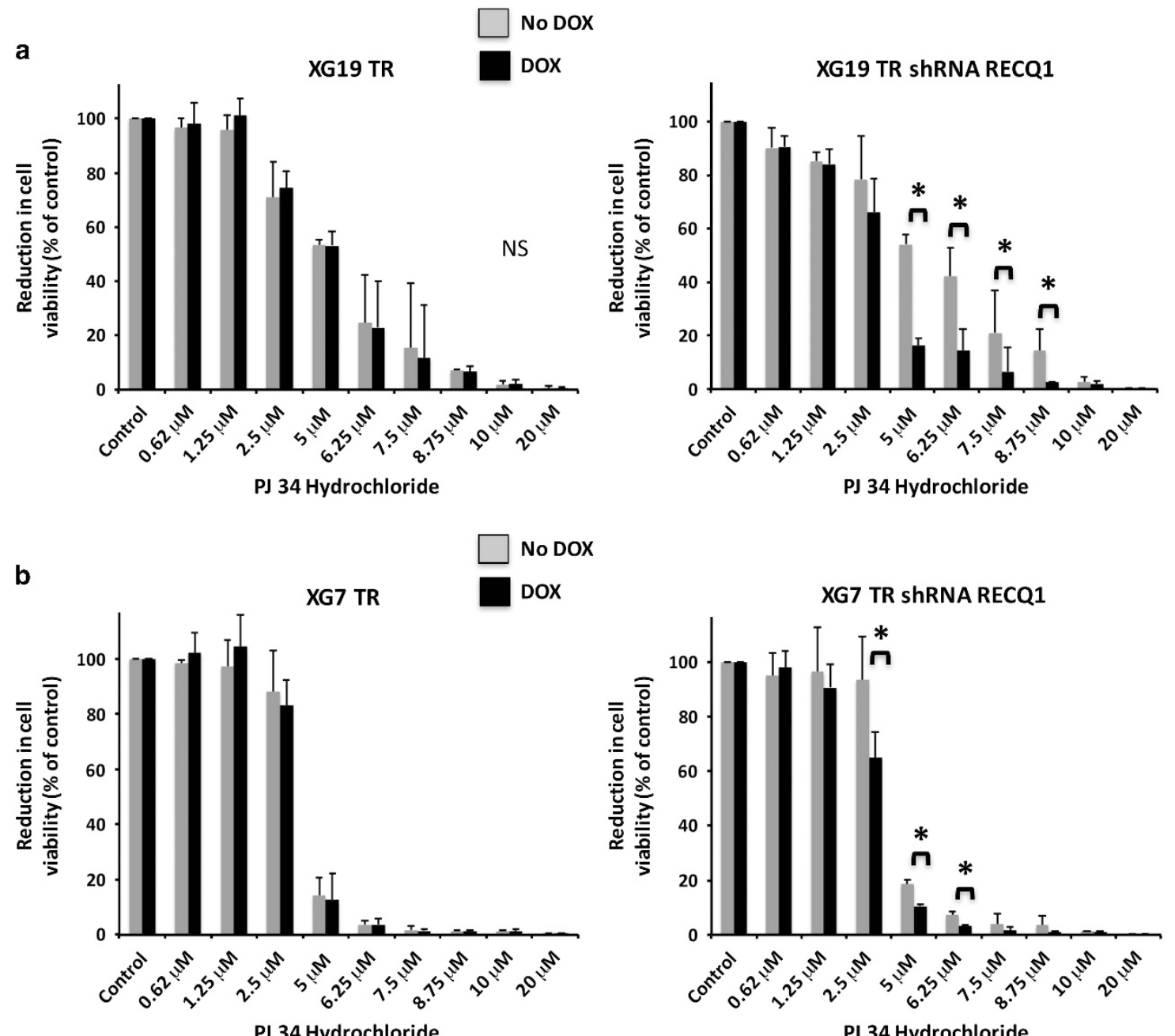

PJ 34 Hydrochloride

PJ 34 Hydrochloride

Figure 6. RECQ1 depletion enhances the sensibility of HMCL to PARP inhibitor. XG19 TR and XG19-TR-shRECQ1 (a) or XG7 TR and XG7 TR shRECQ1 (b) HMCLs were cultured for 4 days in 96-well flat-bottom microtiter plates in RPMI 1640 medium, 10\% fetal calf serum, $2 \mathrm{ng} / \mathrm{ml}$ IL-6 culture medium (control) and graded PJ34 hydrochloride concentrations. Data are mean values \pm s.d. of five experiments determined on sextuplet culture wells. ${ }^{*}$ indicates a significant decrease compared to doxycycline (dox)-untreated cells using a Wilcoxon test for pairs $(P \leqslant 0.05)$.
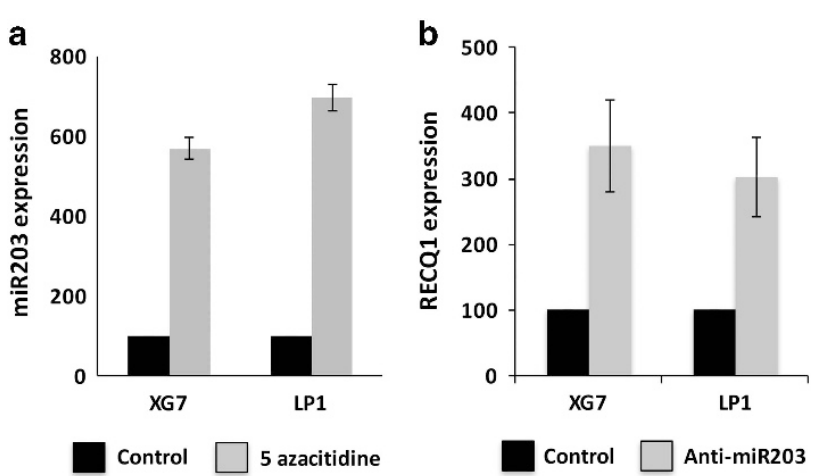

Figure 7. DNMT inhibitor treatment results in RECQ1 downregulation through miR-203 deregulation in MMC. (a) 5-Azacitidine treatment leads to increased miR-203 expression in HMCL. XG7 and LP1 were treated for 7 days with $1 \mu \mathrm{m}$ 5-azacitidine. miR-203 expression levels were determent by RT-qPCR analysis and normalized to miR-16 expression. Data are mean values \pm s.d. of three experiments. (b) miR-203 inhibition results in RECQ1 overexpression in HMCL. XG7 and LP1 cell lines were transfected with anti-miR-203 miRNA inhibitor; RECQ1 expression was assayed by RT-qPCR and normalized by GAPDH expression. Data are mean values \pm s.d. of three experiments. until repair of the Top1 cleavage complex is completed. ${ }^{20}$ These mechanisms could also be involved in $M M C$ resistance to genotoxic agents. Therefore, we reported an increased association of PARP1 and RECQ1 in nuclear extracts of MM cells upon DNA damages induced by melphalan. PARP1 is a target for anticancer therapies and several PARP inhibitors are currently in clinical trials in cancer. ${ }^{57}$ The effects of PARPi were initially linked to inhibition of the role of PARP1 in base excision repair of DNA damages induced by endogenous or exogenous agents, resulting in accumulation of single-strand breaks, which upon conversion to toxic DSB lesions would kill cancer cells deficient in DSB repair. ${ }^{57}$ PARPi could also have a therapeutic activity targeting the role of PARP1 in other DNA repair pathways such as $\mathrm{NER}^{58}$ and also via upregulation of NHEJ pathway leading to increased genomic instability due to imprecise repair. ${ }^{59}$ Our results demonstrate that RECQ1 depletion significantly sensitizes MMCs to PARPi-induced apoptosis. Coupled to the inhibitory effect of PARPi on accumulation of regressed replication forks, it could explain the higher sensitivity of RECQ1-depleted MMCs to PARPi treatment. RECQ1 expression was identified to be inhibited after DNMTi treatment in MM cells. ${ }^{17,60}$ We identified RECQ1 as a target of miR-203 in MM cells, as previously reported in tongue carcinoma. ${ }^{27}$ The aberrant methylation of miR-203 promoter reported in MMCs compared to 

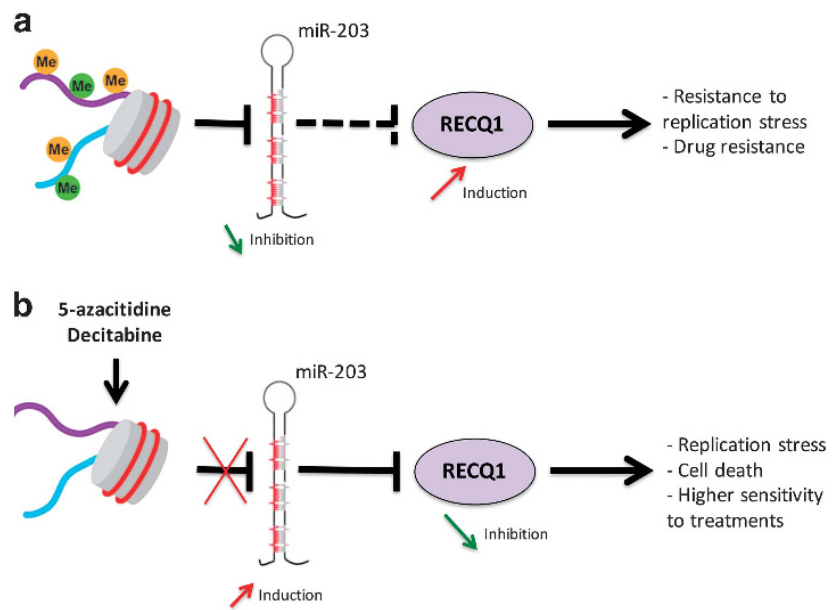

Figure 8. Model of RECQ1 expression regulation and functions in $\mathrm{MM}$ cells. (a) Aberrant methylation of miR-203 is associated with abnormal RECQ1 overexpression in MM cells conferring resistance to replicative stress and treatment. (b) DNMTi treatment induces a significant upregulation of miR-203 expression downregulating RECQ1 expression in MM cells in association with replicative stress, cell death and hypersensitivity to treatments.

normal plasma cells ${ }^{51}$ underlines a mechanism responsible for abnormal RECQ1 overexpression in MM cells (Figure 8). Thus, DNMTi treatment presents therapeutic interest to target RECQ1 expression in $M M$ and induces replicative stress and higher sensitivity to DNA damaging agents (Figure 8 and Supplementary Figure S18). Our data suggest that the association of PARPi to DNMTi and melphalan could act as synthetic lethality treatment in myeloma patients with high expression of RECQ1 and adverse prognosis (Supplementary Figure S20). A study reported that proteasome inhibitor sensitizes MM cells to PARPi treatment. ${ }^{56}$ According to our results demonstrating the protective effect of RECQ1 overexpression against bortezomib therapy and the cooperation between RECQ1 and PARP1 in DNA replication, combination between $\mathrm{DNMTi}$, proteasome inhibitor and PARPi could be of clinical interest in MM. Interestingly, targeted inhibitors against RECQ1 helicase are currently in development. $^{61}$

Our data suggest that combination of DNMTi to target RECQ1 expression with melphalan, proteasome inhibitors and/or PARP inhibitors could have a therapeutic interest in RECQ $1^{\text {high }}$ myeloma patients associated with a poor survival.

\section{CONFLICT OF INTEREST}

The authors declare no conflict of interest.

\section{ACKNOWLEDGEMENTS}

This work was supported by grants from French INCA (Institut National du Cancer) Institute (2012-109/087437 and PLBIO15-256), Languedoc Roussillon CRLR (R14026FF), Fondation de France (201400047510), ITMO Cancer (MM\&TT) and Siric Montpellier (INCa-DGOS-Inserm 6045). EV is supported by a grant from Guillaume Espoir association (Saint-Genis-Laval, France).

\section{AUTHOR CONTRIBUTIONS}

EV performed research and participated in the writing of the paper. $C G, A B$ and CG participated in the research. HG, AS, LV and DH participated in clinical data analysis and participated in the writing of the paper. LT participated in proteomic analyses. $\mathrm{CH}$ supervised proteomic analyses and participated in the writing of the paper. BK, JB, Y-LL, AC and PP participated in the research and in

the writing of the paper. JM supervised the research and the writing of the paper.

\section{REFERENCES}

1 Morgan GJ, Walker BA, Davies FE. The genetic architecture of multiple myeloma. Nat Rev Cancer 2012; 12: 335-348.

2 Walker BA, Wardell CP, Melchor L, Hulkki S, Potter NE, Johnson DC et al. Intraclonal heterogeneity and distinct molecular mechanisms characterize the development of $\mathrm{t}(4 ; 14)$ and $\mathrm{t}(11 ; 14)$ myeloma. Blood 2012; 120: 1077-1086.

3 Magrangeas F, Avet-Loiseau H, Gouraud W, Lode L, Decaux O, Godmer P et al. Minor clone provides a reservoir for relapse in multiple myeloma. Leukemia 2013; 27: 473-481.

4 Weston-Bell N, Gibson J, John M, Ennis S, Pfeifer S, Cezard T et al. Exome sequencing in tracking clonal evolution in multiple myeloma following therapy. Leukemia 2013; 27: 1188-1191.

5 Egan JB, Shi CX, Tembe W, Christoforides A, Kurdoglu A, Sinari S et al. Whole-genome sequencing of multiple myeloma from diagnosis to plasma cell leukemia reveals genomic initiating events, evolution, and clonal tides. Blood 2012; 120: 1060-1066.

6 Keats JJ, Chesi M, Egan JB, Garbitt VM, Palmer SE, Braggio E et al. Clonal competition with alternating dominance in multiple myeloma. Blood 2012; 120: 1067-1076.

7 Lohr JG, Stojanov P, Carter SL, Cruz-Gordillo P, Lawrence MS, Auclair D et al. Widespread genetic heterogeneity in multiple myeloma: implications for targeted therapy. Cancer Cell 2014; 25: 91-101.

8 Chi P, Allis CD, Wang GG. Covalent histone modifications-miswritten, misinterpreted and mis-erased in human cancers. Nat Rev Cancer 2010; 10: 457-469.

9 Issa JP. DNA methylation as a therapeutic target in cancer. Clin Cancer Res 2007; 13: $1634-1637$.

10 Oki Y, Jelinek J, Shen L, Kantarjian HM, Issa JP. Induction of hypomethylation and molecular response after decitabine therapy in patients with chronic myelomonocytic leukemia. Blood 2008; 111: 2382-2384.

11 Moreau P. The future of therapy for relapsed/refractory multiple myeloma: emerging agents and novel treatment strategies. Semin Hematol 2012; 49(Suppl 1): S33-S46.

12 Maes K, Menu E, Van Valckenborgh E, Van Riet I, Vanderkerken K, De Bruyne E. Epigenetic modulating agents as a new therapeutic approach in multiple myeloma. Cancers 2013; 5: 430-461.

13 Dimopoulos M, Siegel DS, Lonial S, Qi J, Hajek R, Facon T et al. Vorinostat or placebo in combination with bortezomib in patients with multiple myeloma (VANTAGE 088): a multicentre, randomised, double-blind study. Lancet Oncol 2013; 14: 1129-1140.

14 San-Miguel JF, Hungria VT, Yoon SS, Beksac M, Dimopoulos MA, Elghandour A et al. Panobinostat plus bortezomib and dexamethasone versus placebo plus bortezomib and dexamethasone in patients with relapsed or relapsed and refractory multiple myeloma: a multicentre, randomised, double-blind phase 3 trial. Lancet Oncol 2014; 15: 1195-1206.

15 Laubach JP, Moreau P, San-Miguel JF, Richardson PG. Panobinostat for the treatment of multiple myeloma. Clin Cancer Res 2015; 21: 4767-4773.

16 Moreaux J, Reme T, Leonard W, Veyrune JL, Requirand G, Goldschmidt H et al. Gene expression-based prediction of myeloma cell sensitivity to histone deacetylase inhibitors. Br J Cancer 2013; 109: 676-685.

17 Moreaux J, Reme T, Leonard W, Veyrune JL, Requirand G, Goldschmidt H et al. Development of gene expression-based score to predict sensitivity of multiple myeloma cells to DNA methylation inhibitors. Mol Cancer Ther 2012; 11: 2685-2692.

18 Chu WK, Hickson ID. RecQ helicases: multifunctional genome caretakers. Nat Rev Cancer 2009; 9: 644-654.

19 Croteau DL, Popuri V, Opresko PL, Bohr VA. Human RecQ helicases in DNA repair, recombination, and replication. Annu Rev Biochem 2014; 83: 519-552.

20 Berti M, Ray Chaudhuri A, Thangavel S, Gomathinayagam S, Kenig S, Vujanovic M et al. Human RECQ1 promotes restart of replication forks reversed by DNA topoisomerase I inhibition. Nat Struct Mol Biol 2013; 20: 347-354.

21 Popuri V, Croteau DL, Brosh Jr RM, Bohr VA. RECQ1 is required for cellular resistance to replication stress and catalyzes strand exchange on stalled replication fork structures. Cell Cycle 2012; 11: 4252-4265.

22 Sharma S, Brosh RM Jr. Unique and important consequences of RECQ1 deficiency in mammalian cells. Cell Cycle 2008; 7: 989-1000.

23 Lu X, Parvathaneni S, Hara T, Lal A, Sharma S. Replication stress induces specific enrichment of RECQ1 at common fragile sites FRA3B and FRA16D. Mol Cancer 2013; 12: 29.

24 Futami K, Kumagai E, Makino H, Goto H, Takagi M, Shimamoto A et al. Induction of mitotic cell death in cancer cells by small interference RNA suppressing the expression of RecQL1 helicase. Cancer Sci 2008; 99: 71-80. 
25 Mendoza-Maldonado R, Faoro V, Bajpai S, Berti M, Odreman F, Vindigni M et al. The human RECQ1 helicase is highly expressed in glioblastoma and plays an important role in tumor cell proliferation. Mol Cancer 2011; 10: 83.

26 Matsushita Y, Yokoyama Y, Yoshida H, Osawa Y, Mizunuma M, Shigeto T et al. The level of RECQL1 expression is a prognostic factor for epithelial ovarian cancer. J Ovarian Res 2014; 7: 107.

27 Tao J, Tao S, Han J, Zhou Z, Zhang X, Wang H et al. RECQL1 plays an important role in the development of tongue squamous cell carcinoma. Cell Physiol Biochem 2014; 33: 1579-1590.

28 Arai A, Chano T, Futami K, Furuichi Y, Ikebuchi K, Inui T et al. RECQL1 and WRN proteins are potential therapeutic targets in head and neck squamous cell carcinoma. Cancer Res 2011; 71: 4598-4607.

29 Futami K, Kumagai E, Makino H, Sato A, Takagi M, Shimamoto A et al. Anticancer activity of RecQL1 helicase siRNA in mouse xenograft models. Cancer Sci 2008; 99: 1227-1236.

30 Li XL, Lu X, Parvathaneni S, Bilke S, Zhang H, Thangavel S et al. Identification of RECQ1-regulated transcriptome uncovers a role of RECQ1 in regulation of cancer cell migration and invasion. Cell Cycle 2014; 13: 2431-2445.

31 Walters DK, Wu X, Tschumper RC, Arendt BK, Huddleston PM, Henderson KJ et al. Evidence for ongoing DNA damage in multiple myeloma cells as revealed by constitutive phosphorylation of H2AX. Leukemia 2011; 25: 1344-1353.

32 Gourzones-Dmitriev C, Kassambara A, Sahota S, Reme T, Moreaux J, Bourquard P et al. DNA repair pathways in human multiple myeloma: role in oncogenesis and potential targets for treatment. Cell Cycle 2013; 12: 2760-2773.

33 Kassambara A, Gourzones-Dmitriev C, Sahota S, Reme T, Moreaux J, Goldschmidt H et al. A DNA repair pathway score predicts survival in human multiple myeloma: the potential for therapeutic strategy. Oncotarget 2014; 5: 2487-2498.

34 Cottini F, Hideshima T, Suzuki R, Tai YT, Bianchini G, Richardson PG et al. Synthetic lethal approaches exploiting DNA damage in aggressive myeloma. Cancer Discov 2015; 5: 972-987.

35 Anderson KC. The 39th David A. Karnofsky Lecture: bench-to-bedside translation of targeted therapies in multiple myeloma. J Clin Oncol 2012; 30: 445-452.

36 Moreaux J, Klein B, Bataille R, Descamps G, Maiga S, Hose D et al. A high-risk signature for patients with multiple myeloma established from the molecular classification of human myeloma cell lines. Haematologica 2011; 96: 574-582.

37 Hose D, Reme T, Hielscher T, Moreaux J, Messner T, Seckinger A et al. Proliferation is a central independent prognostic factor and target for personalized and risk-adapted treatment in multiple myeloma. Haematologica 2011; 96: 87-95.

38 Barlogie B, Tricot G, Rasmussen E, Anaissie E, van Rhee F, Zangari M et al. Total therapy 2 without thalidomide in comparison with total therapy 1: role of intensified induction and posttransplantation consolidation therapies. Blood 2006; 107: 2633-2638.

39 Pineda-Roman M, Zangari M, Haessler J, Anaissie E, Tricot G, van Rhee F et al. Sustained complete remissions in multiple myeloma linked to bortezomib in total therapy 3: comparison with total therapy 2. Br J Haematol 2008; 140: 625-634.

40 Mulligan G, Mitsiades C, Bryant B, Zhan F, Chng WJ, Roels S et al. Gene expression profiling and correlation with outcome in clinical trials of the proteasome inhibitor bortezomib. Blood 2007; 109: 3177-3188.

41 Kassambara A, Schoenhals M, Moreaux J, Veyrune JL, Reme T, Goldschmidt $\mathrm{H}$ et al. Inhibition of DEPDC1A, a bad prognostic marker in multiple myeloma, delays growth and induces mature plasma cell markers in malignant plasma cells. PLoS One 2013; 8: e62752.

42 Zhan F, Huang Y, Colla S, Stewart JP, Hanamura I, Gupta S et al. The molecular classification of multiple myeloma. Blood 2006; 108: 2020-2028.

43 Hothorn $T$, Lausen B. On the exact distribution of maximally selected rank statistics. Comput Stat Data Anal 2003; 43: 121-137.

44 Moreaux J, Cremer FW, Reme T, Raab M, Mahtouk K, Kaukel P et al. The level of $\mathrm{TACl}$ gene expression in myeloma cells is associated with a signature of microenvironment dependence versus a plasmablastic signature. Blood 2005; 106: 1021-1030.
45 Schoenhals M, Frecha C, Bruyer A, Caraux A, Veyrune JL, Jourdan M et al. Efficient transduction of healthy and malignant plasma cells by lentiviral vectors pseudotyped with measles virus glycoproteins. Leukemia 2012; 26: 1663-1670.

46 Schoenhals M, Jourdan M, Seckinger A, Pantesco V, Hose D, Kassambara A et al. Forced KLF4 expression increases the generation of mature plasma cells and uncovers a network linked with plasma cell stage. Cell Cycle 2016; 15: 1919-1928.

$47 \mathrm{Wu}$ Y, Brosh RM Jr. Distinct roles of RECQ1 in the maintenance of genomic stability. DNA Repair (Amst) 2010; 9: 315-324.

48 Rezazadeh S. RecQ helicases; at the crossroad of genome replication, repair, and recombination. Mol Biol Rep 2011; 39: 4527-4543.

49 Parvathaneni S, Stortchevoi A, Sommers JA, Brosh Jr RM, Sharma S Human RECQ1 interacts with Ku70/80 and modulates DNA end-joining of doublestrand breaks. PLoS One 2013; 8: e62481.

50 Veith S, Mangerich A. RecQ helicases and PARP1 team up in maintaining genome integrity. Ageing Res Rev 2015; 23(Pt A): 12-28.

51 Wong KY, Liang R, So CC, Jin DY, Costello JF, Chim CS. Epigenetic silencing of MIR203 in multiple myeloma. Br J Haematol 2011; 154: 569-578.

52 Sharma S. An appraisal of RECQ1 expression in cancer progression. Front Genet 2014; 5: 426.

53 Jackson SP, Durocher D. Regulation of DNA damage responses by ubiquitin and SUMO. Mol Cell 2013; 49: 795-807.

54 Yarde DN, Oliveira V, Mathews L, Wang X, Villagra A, Boulware D et al. Targeting the Fanconi anemia/BRCA pathway circumvents drug resistance in multiple myeloma. Cancer Res 2009; 69: 9367-9375.

55 Jacquemont $C$, Taniguchi T. Proteasome function is required for DNA damage response and fanconi anemia pathway activation. Cancer Res 2007; 67: 7395-7405.

56 Neri P, Ren L, Gratton K, Stebner E, Johnson J, Klimowicz A et al. Bortezomibinduced 'BRCAness' sensitizes multiple myeloma cells to PARP inhibitors. Blood 2011; 118: 6368-6379.

57 Curtin NJ, Szabo C. Therapeutic applications of PARP inhibitors: anticancer therapy and beyond. Mol Aspects Med 2013; 34: 1217-1256.

58 Robu M, Shah RG, Petitclerc N, Brind'Amour J, Kandan-Kulangara F, Shah GM Role of poly(ADP-ribose) polymerase-1 in the removal of UV-induced DNA lesions by nucleotide excision repair. Proc Natl Acad Sci USA 2013; 110: 1658-1663.

59 Patel AG, Sarkaria JN, Kaufmann SH. Nonhomologous end joining drives poly (ADP-ribose) polymerase (PARP) inhibitor lethality in homologous recombinationdeficient cells. Proc Natl Acad Sci USA 2011; 108: 3406-3411.

60 Moreaux J, Bruyer A, Veyrune JL, Goldschmidt H, Hose D, Klein B. DNA methylation score is predictive of myeloma cell sensitivity to 5-azacitidine. $\mathrm{Br} \mathrm{J}$ Haematol 2014; 164: 613-616.

61 Zuo M, Maxwell D, Prasad BAB, Peng Z, Bornmann W, Javle MM. Abstract 5361 development of targeted inhibitors against RecQ1 helicase. Cancer Res 2014; 74 (Suppl 19): 5361.

62 Jourdan M, Caraux A, De Vos J, Fiol G, Larroque M, Cognot C et al. An in vitro model of differentiation of memory $B$ cells into plasmablasts and plasma cells including detailed phenotypic and molecular characterization. Blood 2009; 114: 5173-5181.

(i) $\Theta$ This work is licensed under a Creative Commons AttributionNonCommercial-NoDerivs 4.0 International License. The images or other third party material in this article are included in the article's Creative Commons license, unless indicated otherwise in the credit line; if the material is not included under the Creative Commons license, users will need to obtain permission from the license holder to reproduce the material. To view a copy of this license, visit http:// creativecommons.org/licenses/by-nc-nd/4.0/

(c) The Author(s) 2017

Supplementary Information accompanies this paper on the Leukemia website (http://www.nature.com/leu) 